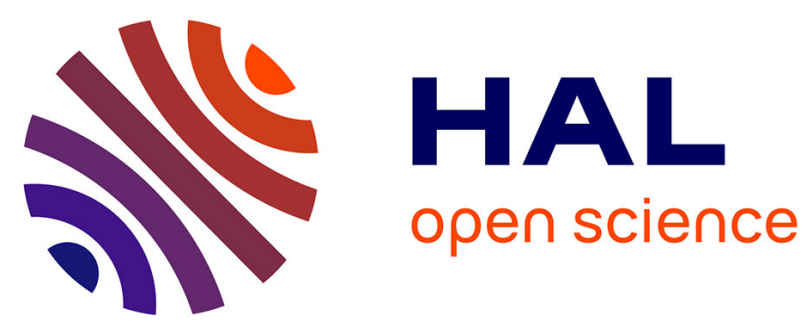

\title{
NUCLEAR GIANT RESONANCES IN COORDINATE SPACE - A SEMICLASSICAL DENSITY FUNCTIONAL APPROACH
}

\author{
P. Gleissl, M. Brack, J. Meyer, Ph. Quentin
}

\section{- To cite this version:}

P. Gleissl, M. Brack, J. Meyer, Ph. Quentin. NUCLEAR GIANT RESONANCES IN COORDINATE SPACE - A SEMICLASSICAL DENSITY FUNCTIONAL APPROACH. International Workshop On Semiclassical And Phase Space Approaches To The Dynamics Of The Nucleus, 1987, Aussois, France. pp.C2-3-C2-10, 10.1051/jphyscol:1987201 . jpa-00226466

\section{HAL Id: jpa-00226466 https://hal.science/jpa-00226466}

Submitted on 1 Jan 1987

HAL is a multi-disciplinary open access archive for the deposit and dissemination of scientific research documents, whether they are published or not. The documents may come from teaching and research institutions in France or abroad, or from public or private research centers.
L'archive ouverte pluridisciplinaire HAL, est destinée au dépôt et à la diffusion de documents scientifiques de niveau recherche, publiés ou non, émanant des établissements d'enseignement et de recherche français ou étrangers, des laboratoires publics ou privés. 


\title{
NUCLEAR GIANT RESONANCES IN COORDINATE SPACE - A SEMICLASSICAL DENSITY FUNCTIONAL APPROACH ( 1 )
}

\author{
P. GLEISSL, M. BRACK, J. MEYER (2) and Ph. QUENTIN (3) \\ Institut für Theoretische Physik, Universität Regensburg, \\ D-8400 Regensburg, F.R.G.
}

\begin{abstract}
Résumé: Dans le cadre d'une description semiclassique de résonnances géantes nucléaires (GR) en utilisant la force SkM* et les fonctionnelles ETF complètes à l'ordre 4 en $\hbar$, nous présentons des modes propres monopolaires $\left(0^{+}\right)$isoscalaires $(I=0)$ et isovectorielles $(I=1)$ en bon accord avec $l^{\prime}$ expérience, ainsi que la variation de quelques énergies GR typiques en fonction de la température.

Abstract: We discuss the semiclassical description of nuclear giant resonances (GR) using a realistic Skyrme force (SkM*) and complete ETF density functionals. We present monopole $\left(0^{+}\right)$eigenmodes of isoscalar $(I=0)$ and isovector $(I=1)$ type, which are in good agreement with experiment, and the corresponding $\mathrm{m}_{1}$ and $\mathrm{m}_{3}$ sum rules. We also present the temperature dependence of some typical GR energies $\left(0^{+}, I=0,1 ; 1^{-}, I=1 ; 2^{+}, I=0\right)$ in $208 \mathrm{pb}$.
\end{abstract}

The density variational method using Skyrme type effective forces and semiclassical ETF (extended Thomas-Fermi) density functionals has been very successful for the calculation of average static properties of nuclei $/ 1,2 /$. This approach has been shown / I/ to yield precisely the average part of selfconsistent microscopical HF (Hartree-Fock) results, leaving out the shell effects. (Similar results were recently obtained also for the Gogny finite-range force D1 $/ 3 /$.) Shell effects in the nuclear binding and deformation energies can be recovered perturbatively to a high degree of accuracy $/ 1,4 /$ by exploiting the strutinsky energy theorem $/ 5,6 /$. The density functional approach becomes particularly gratifying for highly excjted nuclear systems: At excitation energies corresponding to temperatures of $k T \approx 2.5-3 \mathrm{MeV}$ and higher, shell effects vanish and the ETF density functionals become exact $/ 7,8 /$, thus yielding the same results as fully microscopic HF calculations.

When applying these ideas to the description of dynamic nuclear processes, one is at first handicapped by the fact that the ETF functionals do not apply, in general, to situations where the density is time dependent, except for the case of slow adiabatic motion. Although some formal progress has been achieved in the foundation of time-dependent density functional theory $19 /$, ready-to-use functionals are not yet at hand.

In the study of giant resonances (GR) it has been realized /10/ that dynamical deformations of the Fermi sphere in momentum space, which are missed when using the static ETF functionals $/ 11 /$, give important contributions to the restoring forces of some modes (e.g. all isoscalar electric multipole modes with $L \geqslant 2$ ). These effects, which in an infinite system lead to Landau zero-sound excitations, have been incorporated to various degrees of sophistication in the so-called fluid-dynamical approach and its variations $/ 10,12-17 /$, leading to good agreement with the results of RPA calculations and - to the extent that this is the case for the RPA results with experiment. In many of these calculations, however, very schematic energy density functionals ignoring Coulomb and spin-orbit forces were used; the higher-order gradient corrections to the kinetic energy, known to be important to reproduce correctly static nuclear surface and deformation properties $/ 1 /$, were omitted, too.

(1) Work partfally Eupported by the German-Prench aclentiflc exchange program "PROCOPE"

(2) permanent address : I.P.N. (and IN2P3), Universite de Iyon-I, 8-69622 V111eurbanne Cedex, France

(3) permanert addrese : Laboratoire de Physique Theorique, Universite de Bordeaux-I, P-33170 Gradignan. Pranca 
In the present paper we want to report on a related approach which takes full advantage of the complete ETF functionais (also at finite temperatures) and realistic Skyrme interactions in calculating eigenmodes and sum rules corresponding to various giant resonances $/ 18-20 /$. (For similar calculations with approximate ETF functionals see also refs. /21-23/.) As is well-known, there exist theorems /24-26/ relating RPA sum rules to $\mathrm{HF}$ ground state expectation values. In particular, one has (see ref./26/ for detajis)

$$
\begin{aligned}
& m_{3}(Q)=-\frac{1}{2}\langle 0|[\hat{S},[\hat{H}, \hat{S}]]| 0\rangle, \quad \hat{S}=[\hat{H}, Q], \\
& m_{1}(Q)=\frac{1}{2}\langle 0|[Q,[\hat{H}, Q]]| 0\rangle, \\
& m_{-1}(Q)=\frac{1}{2}\left[\frac{d^{2}}{d \lambda^{2}}\langle\lambda|\hat{H}| \lambda\rangle\right]_{\lambda=0}=\frac{1}{2}\left[\frac{d}{d \lambda}\langle\lambda|Q| \lambda\rangle\right]_{\lambda=0} .
\end{aligned}
$$

Hereby $m_{k}(Q)$ is the $k$-th moment of the strength function corresponding to $a$ chosen excjtation operator $Q ;|\lambda\rangle$ is the solution of the static constrained HF problem $\delta\langle\hat{H}-\lambda Q\rangle=0$ with $\lambda$ as a Lagrange multiplier. $\hat{H}$ is the total Hamiltonian of the system including the effective (e.g. Skyrme) nucleon-nucleon interaction. The virtue of eqs. (1)-(3) is that the expressions on the r.h.s. are static HF expectation values which can be evaluated semiclassically with the static ETf functionals (at the possible cost of missing shell effects which, however, are very small for the giant resonance energies, see Fig. 1 below); hereby it is essential that the commutators in eq. (1) be evaluated quantum-mechanically. The knowledge of the three moments (1)-(3) allows one to estimate the mean energy $E$ of a giant resonance peak by the inequality

$$
E_{1} \leqslant E \leqslant E_{3}, \quad E_{k}=\sqrt{m_{k} / m_{k-1}} \text {, }
$$

and its width $\Gamma$ for which $\sqrt{E_{3}^{3}-E_{1}^{2}} / 2$ is an upper bound.

One-body excitation operators $Q$ which commute with the two-body force in $\hat{H}$ (e.g. local and velocity-independent isoscalar operators in connection with Skyrme forces) can be understood as generators of collective scaling-type deformations $\alpha$ by means of unitary transformations of the $\mathrm{HF}$ ground state:

$$
|\alpha\rangle=e^{-\alpha \hat{S}}|0\rangle, \quad \hat{S}=[\hat{T}, Q]=\frac{1}{2} \vec{\nabla} \cdot \vec{u}+\vec{u} \cdot \vec{\nabla}, \quad \vec{u}=-\frac{\pi^{2}}{m} \cdot \vec{\nabla} Q .
$$

Here $\vec{u}$ is recognized as a displacement field defining the velocitv field $\vec{v}=\alpha(t) \vec{u}$ which satisfies the continuity equation $d \rho / c i t+\vec{\nabla} \cdot(\rho \vec{v})=0$, where $\rho(\vec{r}, \alpha(t))$ is the scaled HF ground state density. The moment $m_{1}$ is then easily shown to be proportional to the hydrodynamical inertial parameter $B_{\alpha}$ :

$$
m_{1}(Q)=\frac{m}{2 \hbar^{2}} \int d^{3} r \vec{u}^{2} p=\frac{1}{2 \hbar^{2}} B_{\alpha} \text {, }
$$
and $m_{3}$ is found to be one-half of the harmonic oscillator spring constant $c_{\alpha}$ of the
scaled HF energy:

$$
m_{3}(Q)=\frac{1}{2}\left[\frac{d^{2}}{d \alpha^{2}}\langle\alpha|\hat{H}| \alpha\rangle\right]_{\alpha=0}=\frac{1}{2} c_{\alpha} .
$$

Thus, the energy $E_{3}$ in $(4)$ is equal to the (first) vibrational excitation energy of the collective scaling Hamiltonian

$$
H_{\text {coll }}(\alpha)=\frac{1}{2} B_{\alpha} \dot{\alpha}^{2}+\langle\alpha|\hat{H}| \alpha\rangle
$$

in the harmonic approximation:

$$
E_{3}=\sqrt{m_{3} / m_{1}}=\hbar \sqrt{c_{\alpha} / B_{\alpha}}=\hbar \omega_{\alpha} .
$$

Care should be taken introducing the static semiclassical functionals after taking the derivative $d^{2} / d \alpha^{2}$ in eq. (7); interchanging this order would lead to the hydrodynamical (and thus often the wrong) spring constants $C_{\alpha}$.

The scaling approach can be generalized to a set of coupled modes $\alpha_{i}(i=1,2, \ldots . M)$ 
generated by $M$ operators $Q_{j} / 18,20,27,28 /$. The problem then consists in finding the eigenfrequencies $\omega_{n}$ of the collective Hamiltonian

$$
H_{\operatorname{coll}}=\frac{1}{2} \sum_{i, j=1}^{M} B_{i j} \dot{\alpha}_{i} \dot{\alpha}_{j}+\frac{1}{2} \sum_{i, j=1}^{M} C_{i j} \alpha_{i} \alpha_{j}+E_{H F}
$$

by solving the matrix equation

$$
\left(c-w_{n}^{2} B\right) \vec{x}_{n}=0 \quad(n=1,2, \ldots M)
$$

with

$$
\begin{aligned}
& B_{i j}=2 \hbar^{2} m_{1}(i, j)=\hbar^{2}\left\langle 0\left|\left[Q_{i},\left[\hat{H}, Q_{j}\right]\right]\right| 0\right\rangle, \\
& c_{i j}=2 m_{3}(i, j)=-\left\langle 0\left|\left[\hat{S}_{i},\left[\hat{H}, \hat{s}_{j}\right]\right]\right| 0\right\rangle .
\end{aligned}
$$

Exploiting the generalized Thouless theorem /26/ for the mixed moments

$$
m_{k}(i, j)=\sum_{n>0}\left(\widetilde{E}_{n}-\widetilde{E}_{0}\right)^{k}\left\langle\widetilde{0}\left|Q_{j}\right| \tilde{n}\right\rangle\left\langle\tilde{n}_{\mid}\left|Q_{j}\right| \sigma\right\rangle,
$$

where $|\tilde{n}\rangle$ and $\widetilde{E}_{n}$ are the RPA states and energies, respectively, one can easily show that the $M$ eigenmodes defined by eq.(11) exhaust the $m_{1}$ and $m_{3}$ RPA sum rules eqs.(1), (2) for any operator $Q$ which can be expanded in terms of the finite basis of the $Q_{j}$. (In practice it may be easier - and sometimes more physical - not to start from a guess of the operators $Q_{j}$ but rather to introduce some reasonably chosen collective variables $\alpha_{j}$ and to reconstruct the $\vec{u}_{j}$ and $Q_{j}$ by solving the corresponding continuity equations $/ 18,20,28 /$. )

A difference of the above approach to the fluid-dynamical one (e.g. refs./14,16/) is that in the latter one automatically is led to an infinite set of eigenmodes. This is mathematically satisfying, but has the consequence that many (i.e. almost all) of the eigenmodes tie at energies where the model does not apply (e.g. above the pion threshold) and thus some of the collective strength may appear in unphysical states.

The treatment of isovector $(I=1)$ giant resonances and operators $Q$ is more difficult. The non-zero commutators of $Q$ with the two-body force in this case gives additionalcontributions to the moments $m_{1}$ and $m_{3}$ which are usualiy expressed through the so-called 'acceleration factors' $k_{k}$. Al though these may in principle be incorporated in the scaling approach $/ 12 /$, this becomes rather involved in particular when evaluating $m_{3}(i, j)$ in a multidimensional treatment.

In the following we shall illustrate the above.ideas with a few examples. A more exhaustive presentation of our calculations is in preparation $/ 20 \%$. We used throughout the Skyrme force $S k M * / 1,29 /$ and the full ETF functionals up to fourth order in $\hbar / 1,7,8 /$; so far only spherical nuclei were considered.

In Fig. I we demonstrate the smallness of the shell effects in the energies $E_{3}$ (9) evaluated for some giant resonances as functions of the particle number $A$. The operators $Q$ chosen for calculating $m_{1}$ and $m_{3}$ are

$$
\begin{aligned}
& Q=\sum_{i=1}^{A} r_{i}^{2} \quad \text { for } 0^{+}, I=0 \quad \text { (isoscalar monopole), } \\
& Q=\sum_{i=1}^{A} r_{i}^{2} \tau_{3}(i) \text { for } 0^{+}, I=1 \quad \text { (isovector monopole), } \\
& Q=\sum_{i=1}^{A} r_{i}^{2} P_{2}\left(\cos \theta_{i}\right) \text { for } 2^{+}, I=0 \quad \text { (isoscalar quadrupole). }
\end{aligned}
$$

In the isovector monopole case, the acceleration factors were neglected (see, however, Tab. 2 below). The solid lines show the semiclassical values of $E_{3}$, evaluated with the static ETF functionals for spherical nuclei along a smooth fit to the $B$ stability valley in the $(N, Z)$-plane. They are seen to give an excellent approximation. to the microscopic HF values which are shown by crosses for a sample of corresponding nuclei. (Their shapes were constrained to be spherical in the HF calculations.) We should mention at this place that this close agreement between HF and semiclassical results is not found for the energies $E_{1}$ in all cases; the moments $m_{-1}$ in some modes are much more sensitive to shell effects (see Tab. 2 and the discussion below). 


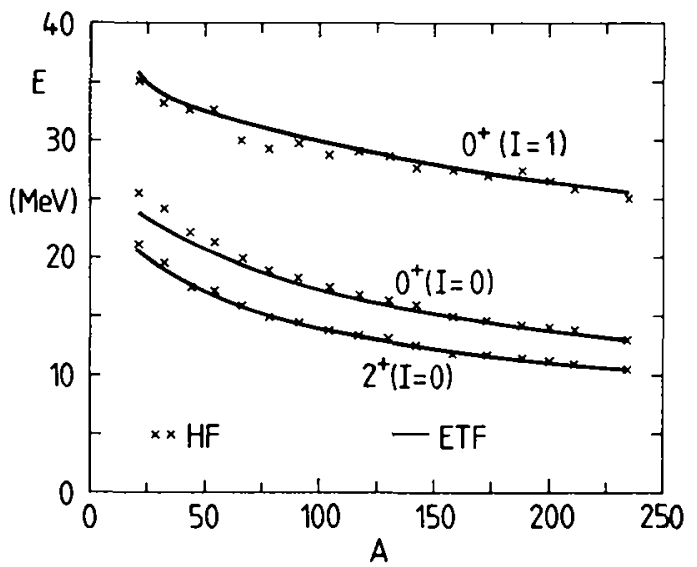

Fig. 1:

GR energies $E_{3}=\sqrt{m_{3} / m_{1}}$ (without acce?eration factors for the $I=1$ monopole) versus nucleon number $A$.

Crosses: HF values, solid line: ETF values for nuclei along the $B$-stability valley in the $(N, Z)$ plane.

Note the small differences which are essentially due to the shell effects. (Minor smooth differences in the light nuclei may also stem from an insufficient parametrization of the nucleon densities in the ETF calculation.) The Skyrme force SkM was used in both calculations.

\begin{tabular}{|c|c|c|c|c|c|c|c|c|}
\hline & \multicolumn{4}{|c|}{$I=0$} & \multicolumn{4}{|c|}{$I=1$} \\
\hline & $\begin{array}{c}\hbar \omega_{1} \\
{[\mathrm{MeV}]}\end{array}$ & $\begin{array}{l}E_{\exp } \\
{[\mathrm{MeV}]}\end{array}$ & $\%\left(m_{1}\right)$ & $x_{0}\left(\mathrm{~m}_{3}\right)$ & $\begin{array}{c}\hbar \omega_{1} \\
{[\mathrm{MeV}]}\end{array}$ & $\begin{array}{l}\mathbf{E}_{\exp } \\
{[\mathrm{MeV}]}\end{array}$ & $\%\left(m_{1}\right)$ & $\%\left(m_{3}\right)$ \\
\hline \multirow{2}{*}{${ }^{40} \mathrm{Ca}$} & 35.5 & & 3.66 & 9.79 & 44.1 & & 19.2 & 34.6 \\
\hline & 21.0 & & 96.34 & 90.21 & 29.5 & $31.1 \pm 2.2$ & 80.8 & 65.4 \\
\hline \multirow{2}{*}{${ }^{60} \mathrm{N1}$} & 33.9 & & 1.23 & 3.59 & 42.6 & & 12.9 & 23.6 \\
\hline & 19.6 & $19.8 \pm 0.5$ & 98.77 & 96.41 & 29.5 & $30.6 \pm 2.3$ & 87.1 & 76.4 \\
\hline \multirow{2}{*}{${ }^{90} \mathrm{zr}$} & 32.1 & & 0.25 & 0.82 & 40.8 & & 7.8 & 14.6 \\
\hline & 17.7 & $16.9 \pm 0.9$ & 99.75 & 99.18 & 28.8 & $28.5 \pm 2.6$ & 92.2 & 85.4 \\
\hline \multirow{2}{*}{${ }^{120} \mathrm{Sn}$} & 30.2 & & 0.05 & 0.17 & 39.5 & & 5.0 & 9.5 \\
\hline & 16.2 & $15.8 \pm 1.0$ & 99.95 & 99.83 & 27.9 & $26.1 \pm 2.4$ & 95.0 & 90.5 \\
\hline \multirow{2}{*}{${ }^{140} \mathrm{Ce}$} & 29.6 & & 0.01 & 0.04 & 38.8 & & 3.8 & 7.2 \\
\hline & 15.4 & $14.8 \pm 0.6$ & 99.99 & 99.96 & 27.5 & $28.0 \pm 2.6$ & 96.2 & 92.8 \\
\hline \multirow{2}{*}{${ }^{208} \mathrm{~Pb}$} & $27 \cdot 5$ & & 0.05 & 0.21 & $37 \cdot 3$ & & 1.5 & 3.1 \\
\hline & 13.5 & $13.7 \pm 0.5$ & 99.95 & 99.79 & 26.0 & $25.5 \pm 3.0$ & 98.5 & 96.9 \\
\hline
\end{tabular}

Tab. 1:

Eigenmode energies $\hbar \omega_{j}(i=1,2)$ of coupled bulk density and surface stiffness monopole $\left(0^{+}\right)$vibrations obtained semiclassically for 6 spherical nuclei with the SKM* force. The experimental GMR peak energies are taken from a compilation in ref. $/ 30 /$ for the isoscalar $(I=0)$ and from ref. $/ 31 /$ for the isovector $(I=1)$ case. Also shown are the percentages of the $m_{1}$ and the $m_{3}$ sum rules for the excitation operators eqs. $(15,19)$. The acceleration factors were neglected in the $I=1$ case.

The idea of the generalized scaling model with coupled collective modes was applied to the giant monopole resonances (GMR, "breathing modes") in refs. $/ 18,20,28 /$. The ground-state densities of neutrons and protons were parametrized by Fermi functions:

$$
\rho_{q}(r)=\rho_{o q} \cdot\left[1+\exp \left\{\left(r-R_{q}\right) / \alpha_{q}\right\}\right]^{-\gamma_{q}} \cdot(q=n, p)
$$

The bulk densities $\rho_{o q}$ and the surface diffuseness parameters $\alpha_{q}$ were taken as collective variables oscillating in time (the radi $\mathrm{R}_{\mathrm{q}}$ being adjusted such as to keep 
the nucieon numbers $N$ and $Z$ constant; the $\gamma_{g}$ are kept constant at the g.s. values). For isoscalar $(I=0)$ vibrations one imposes $\rho_{o n}(t) / \rho_{o n}(0)=\rho_{o p}(t) / \rho_{o p}(0)$, for isovector vibrations $(I=1) \quad Z_{\rho_{o n}}(t) / \rho_{0}(0)=-N \rho_{o p}(t) / \rho_{o p}(0)$, and analogously for the parameters $\alpha_{q}(t)$. Solving eq. (11) gives two eigenmodes in each case. The lower modes are dominated by the bulk density oscillation and can be identified with the experimentally observed GMR peak energies, whereas the higher ones are dominated by the surface oscillation and have not yet been identified experimentally. (Their transition densities typically have two nodes in the surface region $/ 18,20 /$ and are verysimilar to the ones of higher lying $0^{+}$states found also in RPA calculations /D.Gogny, private communication/.)

In Tab. 1 we show the eigenmode energies $\hbar \omega_{j}(i=1,2)$ obtained for some spherical nuclei. The isoscalar results $(I=0)$ correspond to those of ref. $/ 18 /$, but using $\gamma_{\mathrm{g}} \neq 1$ for the g.s. densities (18) in the variational ETF calculation. As already observed in ref. $/ 18 \%$, the agreement with the experimental GMR peak energies (given in Tab. 1 with error bars) is excellent. A similarly good agreement is also found $/ 20 /$ for the lower isovector modes with the newly measured $0^{+}, I=1$ energies $/ 31 /$. This agreement is, however, accidental since the acceleration factors (i.e. the commutators of the operators $Q_{j}$ with the Skyrme force) have been neglected here. Their influence will be discussed in connection with Tab. 2 below. We also show in Tab. 1 the percentages of the $m_{1}$ and $m_{3}$ sum rules for the operator eq. (15) in the $I=0$ and the operator

$$
Q=\frac{A}{4} \sum_{i=1}^{A}\left[\left(\frac{1}{Z}-\frac{1}{N}\right)+\left(\frac{1}{Z}+\frac{1}{N}\right) \cdot \tau_{3}(i)\right] r_{i}^{2} \quad\left(0^{+}, I=1\right)
$$

in the $I=1$ case. (This operator is more correct than that of eq. (16) which leads to a small I=0 component in nuclei with $N \neq Z$.) The distribution of strength over the two modes reflects the importance of the surface component of the oscillations, which is especially pronounced for light nuclei in the isovector modes, due to a larger coupling of the two modes through the off-diagonal matrix elements $B_{12}$ and $C_{12}$. The result of this coupling is to push the eigenfrequencies $\omega_{j}$ further apart from their uncoupled positions $\sqrt{\mathrm{C}_{i j} / \mathrm{B}_{i j}}$. This is demonstrated also in Fig. 2 for the isovector GMR. The dots with error bars and the crosses connected by a dashed line show the experimental GMR peak energies and the lower eigenenergies $\hbar \omega 1$, respectively, from Tabl.

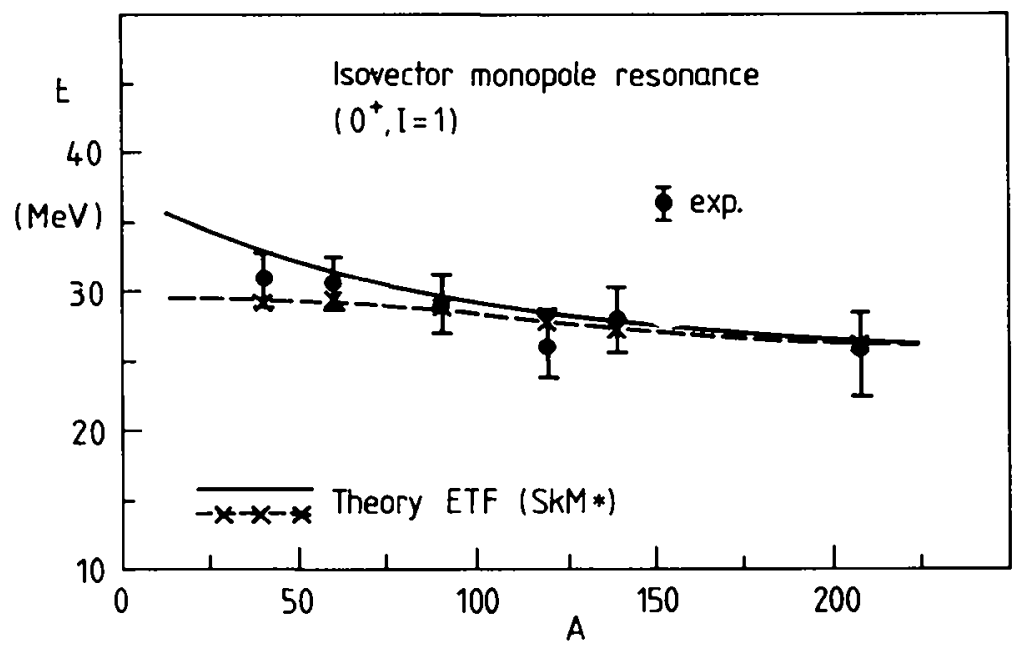

Fig. 2:

Isovector GMR energies obtained in the generalized scaling model (without acceleration factors). Solid line: Energy $E_{3}$ with operator eq. (19). Crosses connected by dashed line: lowest eigenenergy $\hbar \omega_{1}$ of two coupled modes (see text). ETF functionals and SkM* force used. Experimental points from Los Alamos $\left(\pi^{ \pm}, \pi^{0}\right.$ ) measurements $/ 31 /$. 
The solid line results from a one-dimensional semiclassical scaling calculation and corresponds to the energy $E_{3}$ (without acceleration factors) with the operator $Q$ eq. (19). This scaling implies a fixed ratio between surface and bulk density vibrations (ratio $-1 / 3$ between relative changes in $\alpha_{q}$ and $\rho_{o g}$, see ref./18/). Letting the surface vary freely in the two-dimensional calculation lowers the energies $\hbar \omega_{1}$, especially for the lighter nuclei, leading to a somewhat better agreement of their A-dependence with experiment. It would be interesting if experiments for the $I=1$ GMR could be made also for nuclei lighter than $40 \mathrm{Ca}$, since there the predicted differences between the results with one and two collective variables would exceed the expected error bars of the peak energies. The importance of the surface diffuseness degree of freedom for the $0^{+}, I=1$ mode has also been recognized in ref. $/ 32 /$.

\begin{tabular}{|c|cc|cc|cc|}
\cline { 2 - 8 } \multicolumn{1}{c|}{} & \multicolumn{2}{|c|}{$x_{1}$} & $m_{-1}\left[\mathrm{MeV}^{-1} \mathrm{fm}^{4}\right]$ & \multicolumn{2}{|c|}{$\mathrm{E}_{1}$} & {$[\mathrm{MeV}]$} \\
\hline${ }^{40} \mathrm{Ca}$ & 0.251 & 0.242 & 38.7 & 49.4 & 34.7 & 30.7 \\
\hline $90 \mathrm{Zr}$ & 0.295 & 0.297 & 163 & 185 & 32.8 & 30.7 \\
\hline $208 \mathrm{~Pb}$ & 0.333 & 0.332 & 872 & 986 & 29.0 & 27.2 \\
\hline
\end{tabular}

Tab. 2:

Acceleration factors $k_{1}$, sum rules $m_{-1}$ and energies $E_{1}$ for the isovector monopole operator $Q$ eq. (19), calculated for three spherical nuclei with the SkM* force. Both semiclassical (ETF) and Hartree-Fock (HF) results are given.

In. order to check the importance of the acceleration factors neglected so far, we have calculated the full moment $m_{1}$ including the Skyrme force for the isovector monopole operator $Q$ eq. (19). Evaluating the commutators in eq. (2) gives

$$
\begin{aligned}
m_{1} & =\frac{1}{2}\langle 0|[Q,[\uparrow, Q]]| 0\rangle\left(1+\kappa_{1}\right)= \\
& =\frac{A^{2}}{4}\left\{\frac{2 h^{2}}{m}\left(\frac{1}{Z}\left\langle r^{2}\right\rangle_{p}+\frac{1}{N}\left\langle r^{2}\right\rangle_{n}\right)+\left(\frac{1}{Z}+\frac{1}{N}\right)^{2}\left[t_{1}\left(1+\frac{x_{1}}{2}\right)+t_{2}\left(1+\frac{x_{2}}{2}\right)\right] \int r^{2} \rho_{n} \rho_{p} d^{3} r\right\},
\end{aligned}
$$

where $t_{i}$ und $x_{i}$ are Skyrme force parameters. The values of the acceleration factor $k_{1}$ defined in eq. (20) are given in Table 2 for three spherical nuclei. They were evaluated both microscopically (HF) and semiclassically (ETF using the density parametrisation eq. (18)). The differences are $24 \%$ for $\mathrm{Ca}$ and $\mathrm{less}$ than $1 \%$ for medium and heavy nuclei. We also give in Tab. 2 the moments $m_{-1}$ and energies $E_{1}$ from which the $m_{1}$ can easily be gained, see eq. (4). The $m_{1}$ values differ by less than $1 \%$ between ETF and $\mathrm{HF}$ in all nuclei; thus, the $23 \%$ difference in the mean square radii of $\mathrm{Ca} / 1 /$ mostly cancels that in the $\kappa_{1}$ values.

Much larger differences between HF and ETF values are, however, found for the static polarisabilities $m_{-1}$ and thus also for the energies $E_{1}$. These differences reveal a rather strong shell effect in the polarisabilities $\mathrm{m}_{-1}$, amounting to $\sim 22 \%$ in $\mathrm{Ca}$ and $\sim 12 \%$ in the medium and heavy nuclei. It is not too surprising that the response to a static external constraining field, in which the single particle states have time to readjust their orbits, is sensitive to she1r effects. An even more drastic example would be the quadrupole polarisabilities (i.e. the m-1 with $Q$ eq.(17)) of . heavy nuclei, which are well-known to be doninated by the shel1 effects (the semiclassical or liquid drop part only contributing a fraction, see refs. $/ 5,6 /)$.

$\mathrm{HF}$ calculations for the isovector GMR were reported also in ref. $133 \%$. These authors used, however, the excitation operator $Q$ eq. (16). In $40 \mathrm{Ca}$, where there is no difference to the one in eq. (19) which we have used, our HF results for $m_{-1}$ and $E_{1}$ iagree exactly in $N \neq Z$ nuclei they obtain spurious isoscalar $(I=0)$ admixtures amounting e.g. for $208_{\mathrm{Pb}}$ to $-2.3 \mathrm{MeV}$ in $\mathrm{E}_{1}$. 


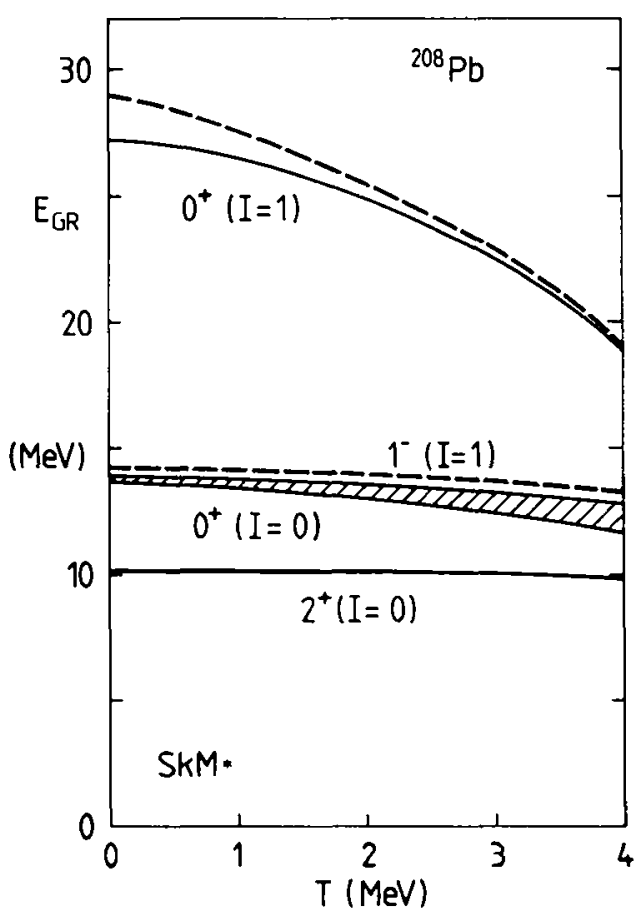

Fig. 3:

Nuclear giant resonance energies versus temperature $T$.

Solid lines: HF results, dashed lines: semiclassical ETF results.

$2^{+}(I=0)$ : energy $E_{3}$ with operator (17).

$0^{+}(I=0)$ : upper line: $E_{3}$, lower line: $E_{1}$ both with operator(15). The difference (=shaded area) is a measure for the width $\Gamma$.

$1^{-}(I=1)$ : energy $E_{1}$ including $\kappa_{1}$ (see ref. /19/ for details).

$0^{+}(I=1)$ : energy $E_{1}$ (including $k_{1}$ ) with operator (19). Note the decrease of the shell effect with increasing temperature.

The SKM* force was used in all cases.

The $H F$ values for $E_{1}$ in Tab. 2 are seen to be in reasonable agreement with the experinental peak energies in Tab. 1, taking the rather large error bars of the latter into account. Note that a similar agreement would be obtained also for the ETF values of $E_{1}$ if the $\kappa_{1}$ factors were neglected; this would, however, be due to a fortuitous cancellation of two completely different errors, namely the omission of $\kappa_{1}$ and of the shell effects in $m_{-1}$.

We finally want to comment briefly on the temperature dependence of the calculated nuclear giant resonance energies. (See also the following paper $/ 23$ / where a more detailed discussion of the finite temperature approach is found.) We limit ourselves here to temperatures up to $\mathrm{T} \sim 4 \mathrm{MeV}(k=1)$ where the continuum effects play a minor role and the density parametrisation eq. (18) is sufficient.

In Fig. 3 we present the energies of various modes as functions of the temperature (see the explanations in the figure caption). They are seen to vary very little (or not at a 17 in the $2^{+}$mode), except for the isovector monopole case $\left(0^{+}, I=1\right)$. Here we give the energy $E_{1}$ using the operator $Q$ eq. (19) and including the $k_{1}$ factor both in HF (solid line) and in ETF (dashed line) approximation. The shell effect discussed above for $T=0$ is seen to decrease with increasing temperature and to practically vanish for $T \geqslant 3 \mathrm{MeV}$, as expected. We do not believe that the isovector GMR is likely to be measured for excited nuclei, but we show these results as another nice example for the disappearance of the sbell effects at $T \geqslant 3 \mathrm{MeV}$ enabling the use of the semiclassical approximation. We refer to ref. /20/ for a more detailed discussion of the finite temperature results and of the dipole $\left(1^{-}\right)$, quadrupole $\left(2^{+}\right)$and octupole $\left(3^{-}\right)$ modes.

We acknowledge stimulating discussions with E.Lipparini, J. and J.P. da Providencia and S.Stringari. Two of US (P.G. and M.B.) acknowledge the warm hospitality of the Institut de Physique Nucleaire at the Universite de Lyon, where part of this work was achieved. 


\section{References:}

1. M. Brack, C. Guet and H.-B. Hakansson, Phys. Reports 123 (1985) 275.

2. J. Treiner and H. Krivine, Ann. of Phys. 170 (1986) 406.

3. J. Meyer et a1., Phys. Lett. 172B (1986) 122.

4. F. Tondeur, A. K. Dutta, J. M. Pearson and R. Behrman, Nucl. Phys. A, to be pub] ished.

5. V. M. Strutinsky, Nucl. Phys. A122 (1968) 1.

6. M. Brack and P. Quentin, Nuc]. Phys. A361 (1981) 35.

7. J. Bartel, M. Brack and M. Durand, Nuc1. Phys. A445 (1985) 263.

8. M. Brack and J. Bartel, Proc. of IVth International Symposium on Recent Progress in Many Body Theories, San Francisco, 1985, eds. P. Siemens and R. Smith (Springer, New York) to be published.

9. H. Kohl, these Proceedings.

10. G. F. Bertsch, Nucl. Phys. A249 (1975) 253.

11. B. K. Jennings, Phys. Lett. $96 \mathrm{~B}$ (1980) 1.

12. S. Stringari, Nucl. Phys. A279 (1977) 454; Ann. Phys. 151 (1983) 35.

13. C. Y. Wong and J. A. MacDona]d, Phys. Rev. C16 (1977) 1196.

14. H. Sagawa and G. Holzwarth, Progr. Theor. Phys. 59 (1978) 1213;

G. Holzwarth and G. Eckardt, Z. Phys. A284 (1978) 291.

15. J. R. Nix and A. J. Sierk, Phys. Rev. C21 (1980) 396.

16. J. P. da Providencia and G. Holzwarth, Nucl. Phys. A398 (1983) 59, A439 (1985) 477 ;

J. P. da Providência, Ph. D. Thesis, Siegen University, 1982.

17. V. M. Strutinsky, A. Magner and V. Denisov, Z. Phys. A315 (1984) 301;

A. Magner and V. M. Strutinsky, Z. Phys. A322 (1985) 633 and preprint 1986.

18. M. Brack and W. Stocker, Nucl. Phys. A406 (1983) 413.

19. J. Meyer, P. Quentin and M. Brack, Phys. Lett. 133B (1983) 279; see also Proc. of 7 th Biennale de Physique Nucléaire, Aussois 1983, Report LYCEN 8302 (Université de Lyon, 1983), p. C131.

20. P. Gleiß1, Diploma Thesis, Regensburg University, 1985;

P. GleiBT, M. Brack, J. Meyer and P. Quentin, to be published.

21. M. Barranco et al., Phys. Lett. $154 \mathrm{~B}$ (1985) 96, and references quoted therein.

22. M. Barranco, A. Polls and J. Martore11, Nucl. Phys. A444 (1985) 445.

23. E. Suraud et al., these Proceedings.

24. D. J. Thouless, Nucl. Phys. 22 (1961) 78.

25. E. R. Marshalek and J. da Providencia, Phys. Rev. C7 (1973) 2281.

26. 0. Bohigas, A. M. Lane and J. Martore11, Phys. Reports 51 (1979) 267.

27. Y. Abgrall et al., Nucl. Phys. A346 (1980) 431.

28. A. S. Jensen and S. M. Larsen, Phys. Scripta 24 (1981) 534.

29. J. Bartel et al., Nucl. Phys. A386 (1982) 79.

30. J. Treiner, H. Krivine, 0. Bohigas and J. Martore17, Nucl. Phys. A371 (1981) 253.

31. A. Erell et al., Phys. Rev. Lett. 52 (1984) 2134.

32. E. Lipparini, these Proceedings, and private communication.

33. M. Casas, J. Martorell and J. Gomez, Phys. Lett. 152B (1985) 6. 